\title{
An epidemic of gastroenteritis due to an uncommon variant of Escherichia coli 0.128
}

\author{
W. I. H. SHEDDEN, C. W. POTTER, V. DUBOWITZ, AND \\ MARGARET WOOD
}

From the Departments of Bacteriology and of Child Health, Sheffield

University, and the Public Health Laboratory, Sheffield

SYNOPSIS A small epidemic of gastroenteritis in a children's hospital is described, and also thei biochemical characteristics of the causative organism. Stress is laid on the fact that failure too recognize that epidemic strains of Escherichia coli may be late lactose fermentors can lead to errorso or delay in diagnosis.

The role of certain enteropathic (epidemic) strains of Escherichia coli in the aetiology of infantile gastroenteritis is now well established. The subject has recently been reviewed by Neter $(1959,1960)$ and by Rogers and Taylor (1961). Adam (1923) suggested that these strains might be recognized by their biochemical properties and was able to isolate them from the faeces of children suffering from gastroenteritis. Bray (1945), who investigated 44 cases of this condition, used serological methods for the identification of the strains which he isolated. From 42 cases he isolated the same serotype which he named $E$. coli var neapolitanum. This organism was later classified serologically by Kauffmann (1947) as $E$. coli 0.111 . Bray noted that 34 of the strains which he isolated fermented lactose whereas eight did not. The eight non-lactose fermenting strains were agglutinated to the end titre by an antiserum prepared against a standard lactose-fermenting strain. Later the same serological type was isolated by numerous workers, including Taylor, Powell, and Wright (1949), who studied eight outbreaks of gastroenteritis in children, involving a total of $\mathbf{1 1 6}$ cases. They isolated 178 epidemic $E$. coli strains, all of which fermented lactose within 24 hours.

The identification of epidemic $E$. coli strains in a busy laboratory is difficult as reliance on the criterion of colonial morphology is unreliable (Charter and Taylor, 1952). Occasionally a strain appears with some peculiar biological characteristic and is not identified by routine laboratory investigation. An epidemic caused by such a strain is reported here.

Received for publication 3 February 1962.
CLINICAL ASPECTS

In July and August 1961 there was an outbreak of gastro-enteritis in the Children's Hospital, Sheffield. Eighte children under the age of 5 months were involved. had been patients in the same ward. As soon as a clinitaib diagnosis of gastroenteritis was made, the child transferred to an isolation unit in another part of thes hospital.

The original source of the organism was never con clusively established but it is reasonable to surmise tha it was introduced by Case 1 . This child had been admitted to another hospital with diarrhoea and vomiting. Stoo culture on several occasions failed to show any pathogen and he was transferred to a cubicle attached to the main ward on 21 July. Cases 2 and 3 were twins aged 3 months who were admitted on 23 July to a cubicle opposite fon adjustment of their feeding régime. They were dischargeo home on 26 July and readmitted to the isolation uni $\bar{B}$. on 2 August. Case 2 had severe diarrhoea and vomiting. for five days before readmission and Case 3 had a milde? illness with loose stools for one day. Case 4 was admitted to the main ward on 9 August with pyloric stenosis whicl? was treated surgically and he was discharged home on 14 August. Four days later he was readmitted to a cubicle in the isolation unit in a moribund state. He had had diarrhoea and vomiting for 24 hours, and had beero vaguely unwell for three days before that. Cases 5 to $\&$ were mildly ill. These children developed diarrhoea op 15 August, 19 August, 20 August, and 21 August re spectively. Cases 5, 6, and 7 were children in the maip ward. Case 8 had been admitted to the cubicle formerlo occupied by Case 1 .

The severity of the illness varied markedly from patien? to patient; Cases 1,2, and 4 were very severely ill, requiringo parenteral fluid replacement. At the other extreme Case $\mathbb{6}$ passed only one or two loose stools. The other four caseo 
were also mild. All were treated with Neomycin by mouth. The clinical response was excellent and all eight recovered.

\section{BACTERIOLOGY}

Rectal swabs taken from each of the eight patients were plated on MacConkey agar and desoxycholate citrate agar, and were inoculated into selenite $F$ broth. The latter was incubated at $37^{\circ} \mathrm{C}$. for 12 hours and then subcultured on desoxycholate citrate agar. All plate cultures were examined after 24 hours' aerobic incubation at $37^{\circ} \mathrm{C}$. and again after 48 hours. 'Pale' colonies (non-lactose fermenting) were examined for fermentation of glucose, lactose, sucrose, mannitol, and dulcitol. Where the sugar fermentation pattern suggested it, non-lactose fermenting isolates were tested for agglutination by polyvalent Salmonella and Shigella antisera. For the identification of epidemic $E$. coli strains three lactosefermenting ('pink') colonies, together with a sweep through the area of thickest growth on the plate, were tested for agglutination by polyvalent epidemic coli 0 antiserum. Final serological identification of epidemic $E$. coli isolates was by the method of Rogers and Taylor (1961).

Rectal swabs taken on admission from Case 1 gave, on MacConkey agar, a growth of typical pink (lactose-fermenting) coliforms together with a few pale colonies also consisting of Gram-negative bacilli. The biochemical characteristics of the latter organism suggested that it was of the paracolon group. It was not agglutinated by either polyvalent Salmonella or Shigella antiserum. Serological examination of the numerous pink (lactose-fermenting) colonies failed to detect epidemic $E$. coli. Hence the aetiology of the condition remained obscure. Rectal swabs from Cases 2 and 3 yielded on MacConkey agar pure growths of a non-lactose fermenting organism with biochemical properties identical with those of the so-called paracolon organism isolated from Case 1. It was found that these isolates were agglutinated by polyvalent epidemic coli 0 antiserum. The organism was finally identified serologically as $E$. coli 0.128 . Serologically identical organisms were isolated from each of the subsequent five patients with gastroenteritis. Though it was never proved that the organism responsible for the illness in
Case 1 was $E$. coli 0.128 , it seems likely that it was and that the epidemic was initiated by it.

The biochemical properties of the organisms isolated from Cases 2 to 8 were investigated more fully. All strains produced acid and gas after 24 hours in glucose, maltose, mannitol, rhamnose, sorbitol, xylose, and trehalose; acid and gas after three to four days in lactose, sucrose, raffinose, and salicin (one strain, from Case 4, fermented lactose in four hours); and acid and gas after eight to 10 days in dulcitol. No strain fermented adonitol. All strains produced indole and gave a positive methyl red reaction. Ammonium citrate was not utilized and the Voges-Proskauer test was negative.

\section{DISCUSSION}

The colonial appearance of epidemic $E$. coli serotypes after 24 or 48 hours' incubation on MacConkey or desoxycholate citrate agar is indistinguishable from that of the normal bowel commensal species. It has therefore become customary to search for the pathogenic varieties by screening lactose-fermenting (pink) colonies by a slide agglutination test with polyvalent epidemic coli 0 antiserum. The bacteriological findings in this outbreak emphasize that epidemic $E$. coli strains may give pale, non-lactose fermenting colonies on a MacConkey plate and ferment lactose so slowly that they may be designated non-lactose fermenters in a routine laboratory. Failure to appreciate the possible significance of these pale colonies may result in serious delay in diagnosis.

We are grateful to Professor R. Illingworth for permission to study his cases, to Professor C. P. Beattie and Dr. E. H. Gillespie for their interest and advice, and to Dr. Joan Taylor of the Salmonella Reference Laboratory, Colindale, for confirming the identity of the strain.

\section{REFERENCES}

Adam, A. (1923). Jb. Kinderheilk., 101, 295.

Bray, J. (1945). J. Path. Bact., 57, 239.

Charter, Ruth, E., and Taylor. Joan (1952). J. Path. Bact., 64. 729,

Kauffmann, F. (1947). J. Immunol., 57, 71.

Neter, E. (1959). J. Pediat., 55, 223.

- (1960). Pediat. Clin. N. Amer., 7, 1015.

Rogers, K. B., and Taylor, Joan (1961). Bull. Wld Hlth Org., 24. 59.

Taylor, J., Powell, B. W., and Wright, J. (1949). Brit. med. J., 2, 117. 